\title{
Belief Merging and Judgment Aggregation in Fuzzy Setting
}

\author{
Ismat Beg' and Nabeel Butt ${ }^{2}$ \\ ${ }^{1}$ Department of Mathematics and Statistics, Faculty of Management Studies, University of Central Punjab, Lahore, Pakistan \\ ${ }^{2}$ University of Western Ontario, London, ON, Canada N6A $3 \mathrm{~K} 7$
}

Correspondence should be addressed to Ismat Beg, ibeg@lums.edu.pk

Received 12 April 2012; Accepted 28 May 2012

Academic Editor: Rustom M. Mamlook

Copyright () 2012 I. Beg and N. Butt. This is an open access article distributed under the Creative Commons Attribution License, which permits unrestricted use, distribution, and reproduction in any medium, provided the original work is properly cited.

We explore how judgment aggregation and belief merging in the framework of fuzzy logic can help resolve the "Doctrinal Paradox." We also illustrate the use of fuzzy aggregation functions in social choice theory.

\section{Introduction}

Social choice theory defines "preference aggregation" as forming collective preferences over a given set of alternatives. Likewise, “judgment aggregation" pertains to forming collective judgments on a given set of logically interrelated propositions. This paper extends beyond classical propositional logic into the realm of general multivalued logic, so that we can handle realistic collective decision problems (see Dietrich and List [1, 2], List [3], Beg and Butt [4], and Manzini and Mariotti [5]). List and Pettit [6, 7] were the first to give an axiomatic treatment to the problem associated with judgment aggregation. In their classic example, a set of propositions is expressed in propositional calculus as $\{p, q, p \cap q\}$. The set $L=\{(0,0,0),(0,1,0),(1,0,0),(1,1,1)\}$ consists of all assignments of 0 or 1 to the propositions in $\{p, q, p \cap q\}$ that are logically consistent. A procedure for $n$ judges to decide on the truthfulness of each proposition in $\{p, q, p \cap q\}$ amounts to an aggregator that maps $L^{n} \rightarrow L$. The "Doctrinal Paradox" illustrates that proposition-wise majority rule leads to inconsistent collective decisions. This paradox has made the literature on "judgment aggregation" grow appreciably. Most of the discussions on this paradox have been in the domain of social choice theory, and a number of "(im)possibility theorems," similar to those of Arrow [8] and Sen [9] have been proved. In fact, these theorems show that there cannot exist any judgment aggregation procedure that simultaneously satisfies certain minimal consistency requirements (see Dietrich [10]). List and Pettit [6] have shown that the majority rule is but one member of a class of aggregation procedures that fails to ensure consistency in the set of collective judgments. Van Hees [11] has further generalized the paradox by showing that there is even a larger class of aggregation procedures for which this is true.

The aim of this paper is to resolve the paradox and also to illustrate optimal judgment aggregation. We abandon the assumption that individual and collective beliefs necessarily have a binary nature (true or false) and so our analysis is in a fuzzy logic framework. Pigozzi [12] discusses a possibility result in binary logic in which the paradox is avoided at the price of "indecision." Distance-based aggregation procedures like that of Pigozzi [12] often result in dictatorship. Accordingly, aggregation procedures in fuzzy logic can help us make the collective judgment set more "democratic" in nature. In this paper we try to give the present literature in this area a more realistic touch by using fuzzy logic. The structure of the paper is as follows: Section 2 illustrates an example of the "Doctrinal Paradox" and its reformulation in a fuzzy setting. Section 3 illustrates how the paradox is resolved in a fuzzy framework to find optimal fuzzy aggregation functions. Section 4 further elaborates on the results in Section 3 to present a democratic fuzzy aggregation function. Section 5 presents the entire previous discussion in utility maximization framework.

\section{The Doctrinal Paradox}

The doctrinal paradox can emerge when the members of a group have to make a judgment (in the form of yes 
or no) on several logically interconnected propositions, and the individually logically consistent judgments need to be combined into a collective decision. For example, consider a set of propositions, where some (the "premises") are taken to be equivalent to another proposition (the "conclusion"). When majority voting is applied to premises, it may give a different outcome than majority voting applied to conclusion. Suppose that three customers have to decide what response a product launch by a multinational will receive. According to the company, if the price is good (proposition $P$ ) and product is attractive (proposition $Q$ ), then the customer likes the product (proposition $R$ ). Now assume that each customer makes a consistent judgment over these propositions $P, Q$, and $R$ as in Table 1 .

Each customer assigns a binary truth value to the propositions $P, Q$, and $R$ which gives rise to the doctrinal paradox. The paradox lies precisely in the fact that the two procedures may lead to contradictory results depending on whether the majority is taken on the individual judgments of $P$ and $Q$, or whether the majority is calculated on the individual judgments of $R$.

Arguably, in some decision problems, propositions are "vague" and hence can have truth values between "true" and "false." This might be so for "the economy is in a good shape," as "in a good shape" is not precisely defined. To account for vagueness, one might use a fuzzy logic framework.

Let us reformulate the entire problem in a fuzzy logic framework, so that individual judgments can take values on the interval $[0,1]$. In this context " $\wedge$ " is replaced by the fuzzy Lukasiewicz $t$-norm $\Delta$ given by $x \Delta y=0 \vee(x+y-$ $1)$. The ordinary implication " $\rightarrow$ " is replaced by the fuzzy Lukasiewicz implication $\Rightarrow$ which is defined as follows:

$$
x \Longrightarrow y= \begin{cases}1-x+y & \text { if } x>y \\ 1 & \text { if } x \leq y .\end{cases}
$$

One important property that our fuzzy aggregation operator satisfies in this problem is $\min (0.5,0.2,0.8) \leq$ $\theta^{1} \leq \max (0.5,0.2,0.8), \min (0.6,0.7,0.1) \leq \theta^{2} \leq$ $\max (0.6,0.7,0.1)$, and $\min (0.5,0.2,0.1) \leq \theta^{3} \leq$ $\max (0.5,0.2,0.1)$ in Table 2 .

At the same time, let $\pi(P)$ denote the degree of truth of the proposition $P$, and the fuzzy integrity constraint is $\{\pi(P) \Delta \pi(Q) \Rightarrow \pi(R)\}$. Assuming that the customers are "rational," they never violate the fuzzy integrity constraints (see List [13]). Now by using the above given Lukasiewicz $t$-norm and fuzzy implication $\Rightarrow$, the constraint can be translated as $\pi(R) \geq \max (0, \pi(P)+\pi(Q)-1)$. Here $\pi(R)=$ $f_{i}(\pi(P), \pi(Q))$ is a particular rule of inference for individual $i$ (see Claussen and Roisland [14]).

\section{The Doctrinal Paradox and Belief Merging in Fuzzy Framework}

Given a finite set of $n$ individuals, a finite set $X$ of propositions over which individuals have to make their judgments is called an agenda. A judgment set $A_{i}$ for an individual $i$ is an $n$-tuple containing degree of truth for each proposition. Let $A_{i}=\left(h_{i 1}, h_{i 2}, \ldots, h_{i|X|}\right),|X|$ denote the cardinality
TABLE 1

\begin{tabular}{lcccc}
\hline & $P$ & $Q$ & $(P \wedge Q) \rightarrow R$ & $R$ \\
\hline Customer 1 & Yes & Yes & Yes & Yes \\
Customer 2 & Yes & No & Yes & No \\
Customer 3 & No & Yes & Yes & No \\
Majority & Yes & Yes & Yes & $?$ \\
\hline
\end{tabular}

TABle 2

\begin{tabular}{lcccc}
\hline & $P$ & $Q$ & $R$ & $(P \Delta Q) \Rightarrow R$ \\
\hline Customer 1 & 0.5 & 0.6 & 0.5 & 1 \\
Customer 2 & 0.2 & 0.7 & 0.2 & 1 \\
Customer 3 & 0.8 & 0.1 & 0.1 & 1 \\
Majority & $\theta_{1}$ & $\theta_{2}$ & $\theta_{3}$ & 1 \\
\hline
\end{tabular}

of $X$. A profile is an $n$-tuple $\left(A_{1}, A_{2}, \ldots, A_{n}\right)$ of individual judgment sets. An aggregation is a function $f$ that assigns to each profile $\left(A_{1}, A_{2}, \ldots, A_{n}\right)$ a collective judgment set $\left(h^{*}{ }_{1}, h^{*}{ }_{2}, \ldots, h^{*}|X|\right)=f\left(A_{1}, A_{2}, \ldots, A_{n}\right)$. Here $0 \leq h_{j}^{*} \leq 1$ for $j \in\{1,2,3, \ldots,|X|\}$. Let us denote $f\left(A_{1}, A_{2}, \ldots, A_{n}\right)(\varphi)$ as the truth value of some proposition $\varphi$ for the collective judgment set $f\left(A_{1}, A_{2}, \ldots, A_{n}\right)$. Similarly, $A_{i}(\varphi)$ is the truth value of some proposition $\varphi$ for the judgment set $A_{i}$.

Define $f$ as a "dictatorship" if $f\left(A_{1}, A_{2}, \ldots, A_{n}\right)=A_{i}$ for some $i \in\{1,2,3, \ldots, n\}$ and every $\left(A_{1}, A_{2}, \ldots, A_{n}\right)$.

We define $f$ as "manipulable" if and only if there exists some voter $i$, proposition $\varphi$, and profile $\left(A_{1}, A_{2}, \ldots, A_{n}\right)$ such that $A_{i}(\varphi) \neq f\left(A_{1}, A_{2}, \ldots, A_{i}, \ldots, A_{n}\right)(\varphi)$ but $A_{i}(\varphi)=$ $f\left(A_{1}, A_{2}, \ldots, A_{i}^{*}, \ldots, A_{n}\right)(\varphi)$ for some alternate judgment set $A_{i}^{*}$.

We define $f$ as "independent" if and only if for all propositions $\varphi$ there is a function $g_{\varphi}:[0,1]^{n} \rightarrow[0,1]$ such that for all $\left(A_{1}, A_{2}, \ldots, A_{n}\right)$ we have

$$
f\left(A_{1}, A_{2}, \ldots, A_{n}\right)(\varphi)=g_{\varphi}\left(A_{1}(\varphi), A_{2}(\varphi), \ldots, A_{n}(\varphi)\right) .
$$

Belief merging formally investigates how to aggregate a finite number of belief bases into a collective one. This formal framework consists of a propositional language $\chi$ which is built up from a finite set $P$ of propositional letters standing for atomic propositions and the usual connectives $(\neg, \Delta, \nabla, \Rightarrow, \Leftrightarrow)$. These are the connectives in fuzzy logic, namely, fuzzy negation $\neg, t$-norm $\Delta, t$-conorm $\nabla$, and fuzzy implication $\Rightarrow$ (see Nguyen and Walker [15]). Let the belief base $K_{i}$ for the agent $i$ be the following set $\left(\pi_{i}\left(p_{1}\right), \pi_{i}\left(p_{2}\right), \ldots, \pi_{i}\left(p_{|P|}\right)\right),|P|$ denotes the cardinality of $P$. Here $\pi(\cdot)$ represents the truth function that maps elements in set $P$ to $[0,1]$. A belief set is the set $E=\left\{K_{1}, K_{2}, \ldots, K_{n}\right\}$. Given a set of integrity constraints IC in a fuzzy framework, $\psi$ maps $E$ and IC into a new (collective) belief base $\psi_{\mathrm{IC}}(E)$. We call this process fuzzy aggregation.

An interpretation is a function from $P$ to $[0,1]$. Let $W$ denote the set of all interpretations. A distance between interpretations is a real-valued function $d: W \times W \rightarrow \mathfrak{R}$ such that for all $w, w^{\prime}, w^{\prime \prime} \in W$ as follow:

(1) $d\left(w, w^{\prime}\right) \geq 0$,

(2) $d\left(w, w^{\prime}\right)=0$ if and only if $w=w^{\prime}$, 
(3) $d\left(w, w^{\prime}\right)=d\left(w^{\prime}, w\right)$,

(4) $d\left(w, w^{\prime \prime}\right) \leq d\left(w, w^{\prime}\right)+d\left(w^{\prime}, w^{\prime \prime}\right)$.

One possible choice for distance function is the following Euclidean metric:

$$
d^{*}\left(w, w^{\prime}\right)=\left(\sum_{\forall x \in P}\left|w(x)-w^{\prime}(x)\right|^{L}\right)^{1 / L},
$$

for some real number $L \geq 1$.

Now let us define a belief merging operator in the fuzzy framework which helps us avoid the "Doctrinal Paradox." For any interpretation $w \in W$ and any profile of belief basis $K \in$ $\kappa^{n}$, the distance between an interpretation and a profile can now be defined as

$$
D^{d}(w, K)=\sum_{\forall i} d\left(w, K_{i}\right)
$$

Our objective is to choose $w$ which minimizes this distance and also does not violate any integrity constraint in the fuzzy framework. The distance minimization procedure seeks to minimize a measure of "disagreement" in the society by bringing the collective judgment set as close as possible to the individual judgment sets. We believe that individual "disagreement" brings about individual disutility. Accordingly, we seek to minimize the societal disutility which is assumed to be the sum of individual disutilities. For this purpose any deviation of the collective judgment from the individual judgment has a penalty in our objective function. We concede that a wide variety of distance and dissimilarity measures exist like the Manhattan distance, Chebyshev distance, Jaccard dissimilarity, Yule dissimilarity, and so forth. We have chosen Euclidean distance only for the sake of illustration in this paper. Choosing any particular distance or dissimilarity measure is solely at our discretion provided that it satisfies certain normative principles. See Section 5 to view our distance minimization procedure in a utility maximization framework.

Let $w$ be any arbitrary interpretation. In this case, $w(P)=$ $\left(\theta_{1}, \theta_{2}, \ldots, \theta_{|P|}\right)$ where we have $0 \leq \theta_{i} \leq 1$ and $|P|$ denotes the cardinality of $P$. We can now show that if $d$ is Hamming distance then the doctrinal paradox can be avoided in a binary logic framework (see Pigozzi [12]). In this case, $d^{*}$ is a generalization of Hamming distance, and we can use it in a fuzzy framework to help us avoid the doctrinal paradox. By conforming to democratic values, we can formulate the fuzzy aggregation as an optimization problem which can be stated as follows.

Minimize $\quad D^{d}(w, K)=\sum_{\forall i} d^{*}\left(w, K_{i}\right)$,

subject to the fuzzy integrity constraints IC.

Here, consider that

$$
\begin{gathered}
w(P)=\left(\theta_{1}, \theta_{2}, \ldots, \theta_{|P|}\right), \\
\min \left\{K_{1}(j), K_{2}(j), \ldots, K_{n}(j)\right\} \leq \theta_{j} \\
\leq \max \left\{K_{1}(j), K_{2}(j), \ldots, K_{n}(j)\right\}, \\
0 \leq \theta_{j} \leq 1 \text { for } j \in\{1,2,3, \ldots,|P|\},
\end{gathered}
$$

where $K_{i}(j)$ denotes the $j$ th element of the belief base $K_{i}$.
The above optimization problem helps us avoid doctrinal paradox, and we can also find an optimal fuzzy aggregation function. We say that an aggregation function is optimal if the collective judgment set is as close as possible to the individual judgments. Finding collective social choice function in Table 2 now becomes an optimization problem which can have multiple optimal solutions. The problem in Table 2 is framed in Mathematica language assuming that $L=2$ in $d^{*}$. The optimal fuzzy aggregation function gives the solution for Table 2 as $\left(\theta_{1}, \theta_{2}, \theta_{3}\right)=(0.466797,0.555718,0.369934)$. The fact that there is at least one solution to the problem shows that doctrinal paradox cannot occur in this case. In fact, the paradox is resolved, not at the price of "indecision" or "dictatorship" (see Pigozzi [12]).

Ideally we would like our aggregation procedure to be strategy proof. Dietrich and List [16] prove impossibility theorems similar to the Gibbard-Satterthwaite theorem on strategy-proof aggregation rules. Given these theorems we do not claim that our liberal distance-based aggregation procedure is strategy proof. In fact, Dietrich [10] has proved that independence and monotonicity are two properties of an aggregator that result in strategy proofness. Since we do not claim that our distance-based aggregator is independent and monotone simultaneously, the strategy proofness of our aggregator is unclear. However, the nature of our objective function in the optimization problem is such that if an individual was to submit an insincere judgment (in an attempt to manipulate the collective judgment), any deviation of the collective judgment set from this insincere judgment has a penalty in the objective function. In this sense there appears to be a "partial" corrective mechanism whereby our aggregation procedure is not easily prone to manipulation.

Now consider Table 3. For simplicity assume that there is a "small" economy with three individuals, and $X=\{x, y, z\}$ represents three goods. The individual binary relations over $X=\{x, y, z\}$, namely, $\rho_{1}, \rho_{2}$, and $\rho_{3}$ are linear orders. Any optimal fuzzy social preference aggregation function must map individual preferences into social preference set that must be a linear order. This accordingly becomes an optimization problem in which we minimize the sum of the distances of social preference from the individual preferences using $d^{*}$ (as defined earlier subject to fuzzy integrity constraint of linear order). Here preference aggregation is modeled as a case of judgment aggregation by representing preference orderings as truth values in fuzzy logic. Suppose that the individuals have the following preference structure.

The problem in Table 3 is framed in Mathematica language assuming that $L=2$ in $d^{*}$. The optimal fuzzy preference function gives the solution for Table 3 as $\left(\theta_{1}, \theta_{2}\right.$, $\left.\theta_{3}, \theta_{4}, \theta_{5}, \theta_{6}\right)=(0.688307,0.274744,0.351543,0.13985$, $0.54247,0.222919)$. Note, however, that the optimal solution is not necessarily unique.

We admit that our aggregation procedure suffers from nonuniqueness problem (in other words "indecision") whereby the aggregation function could become set valued. Yet it is an improvement upon Pigozzi's [12] aggregation procedure (defined in the context of binary logic) because cases of dictatorship are highly unlikely in our procedure. In fact, we could devise an appropriate tie-breaking procedure 
TABLE 3

\begin{tabular}{ccccccc}
\hline & $x>y$ & $y>x$ & $y>z$ & $z>y$ & $x>z$ & $z>x$ \\
\hline$\rho_{1}$ & 0.5 & 0.2 & 0.5 & 0.1 & 0.4 & 0.2 \\
$\rho_{2}$ & 0.8 & 0.7 & 0.2 & 0.1 & 0.5 & 0.3 \\
$\rho_{3}$ & 0.8 & 0.1 & 0.3 & 0.2 & 0.7 & 0.2 \\
$\rho_{S}$ & $\theta_{1}$ & $\theta_{2}$ & $\theta_{3}$ & $\theta_{4}$ & $\theta_{5}$ & $\theta_{6}$ \\
\hline
\end{tabular}

in case our optimal solution is not unique. Suppose we have a judgment set $\bar{C}=\left(\right.$ average $\left(\pi_{i}\left(p_{1}\right)\right), \ldots$, average $\left.\left(\pi_{i}\left(p_{|P|}\right)\right)\right)$. Such a set $\bar{C}$ might violate the fuzzy integrity constraints. A tie-breaking procedure would narrow down solutions by picking solutions which are at a minimal distance from $\bar{C}$. Another useful procedure would be to select an aggregation function from the optimal solution which is at minimal disagreement with other aggregation functions using appropriate dissimilarity and distance measures. However, we must confess that such a remedy does not ensure nonuniqueness of our final solution. An appropriate social welfare function can be useful in such cases.

The important question is how well behaved is our aggregation operator. On the one hand, we want the collective judgment to be responsive to the judgment of individuals. On the other hand, we want the collective judgment to obey rationality constraints. We note that our fuzzy aggregation procedure satisfies social axioms like unanimity (Pareto conditions), compensativeness, anonymity, nondictatorship, universal domain, and collective rationality. Other properties like monotonicity and citizen sovereignty are, however, unclear. It is worthy to compare our aggregation operator to operators in the fuzzy aggregation. For example, fuzzy LAMA operator (see Peláez and Doña [17]) has unrestricted domain, anonymity, monotonicity, unanimity, and citizen sovereignty. Ironically, it does not ensure collective rationality. Yager [18] has introduced order weighted averaging (OWA) operators which are idempotent, monotone, neutral, and compensative and yet again do not ensure collective rationality.

\section{Democratic Fuzzy Aggregation Function}

The task of aggregating judgments arises in many situations like promotion committees, corporations complying with shareholders, governments bound to party's principles, and so forth. In each case we can think of a collective judgment set $\bar{F}$ which is the weighted average of individual judgment sets. The closer our collective judgment set $F$ is to $\bar{F}$ we believe the more legitimate and democratic is our final judgment. In other words the decision making group is displaying a degree of "integration" (see List and Petit [19]). There can be different possible interpretations for the term "closeness." The interpretation used in our paper is solely for the purpose of illustration and is by no means exhaustive.

A democratic fuzzy aggregation imparts "anonymity" to ensure that all individuals have equal weight in determining collective sets of judgments. Define a particular fuzzy averaging operator as follows:

$$
\operatorname{average}\left(x_{1}, x_{2}, x_{3}\right)=\frac{w_{1} x_{1}+w_{2} x_{2}+w_{3} x_{3}}{w_{1}+w_{2}+w_{3}},
$$

where $w_{i}$ is the weight assigned to the truthfulness of $x_{i}$.

Similarly, we can apply the same averaging operator to individual profiles as

$$
\operatorname{average}\left(A_{1}, A_{2}, A_{3}, \ldots, A_{n}\right)=\frac{\sum_{i=1}^{n} w_{i} A_{i}}{\sum_{i=1}^{n} w_{i}} .
$$

Now we assert that an optimal fuzzy aggregation function is "democratic" if the solution is as close as possible to the average of individual judgments. Such a view is held only for the purposes of illustration in our paper, and there could be different possible ways for "democratization." Consider Table 1. Assume that each customer gives an equally truthful judgment, we can calculate the average of individual judgments as follows:

$$
\begin{aligned}
& \text { average }(0.5,0.2,0.8)=0.500, \\
& \text { average }(0.6,0.7,0.1)=0.4667, \\
& \text { average }(0.5,0.2,0.1)=0.267
\end{aligned}
$$

A decision-making group is exposed to "rationality challenge" and "knowledge challenge" whenever it is appropriate to "personify" it (see List and Pettit [19]). The point (0.500, 0.4667 , and 0.267 ) is itself not used as a solution because it might violate the fuzzy integrity constraints.

A fuzzy aggregation function is democratic as well as optimal if we make the solution of Table 1 as close as possible to the point $(0.500,0.4667$, and 0.267$)$. This could be easily achieved by adding the following penalty to the objective function:

$$
\delta\left(\left(\theta_{1}-0.5\right)^{2}+\left(\theta_{2}-0.467\right)^{2}+\left(\theta_{3}-0.267\right)^{2}\right)^{0.5} .
$$

Here $\delta \in \mathfrak{R}$ is the degree of democracy.

The problem, in Table 2, can be "democratized" in Mathematica language assuming that $L=2$ in $d^{*}$. In this case, the optimal fuzzy aggregation function gives the solution as $\left(\theta_{1}, \theta_{2}, \theta_{3}\right)=(0.5,0.467,0.267)$. It remains a good exercise to "democratize" the optimal social preference aggregation function for Table 3.

\section{Optimal Judgment Aggregation Viewed as a Fuzzy Utility Maximization}

We have assumed that agents have only "epistemic" preferences; that is, they only care about the "distance" between the collective judgment set which is collectively adopted and the individual judgment set they personally favor (see Van Hees [11]). This distance was originally measured as

$$
d^{*}\left(w, w^{\prime}\right)=\left(\sum_{\forall x \in P}\left|w(x)-w^{\prime}(x)\right|^{L}\right)^{1 / L},
$$


for any real number $L \geq 1$, where $P$ is a finite set of propositional letters standing for atomic propositions. We rescale this distance to form a new distance measure as follows

$$
\tilde{d}(p, q)=\frac{d^{*}(p, q)}{|P|} .
$$

Now this rescaling makes $\tilde{d}(p, q) \in[0,1]$. Let $p_{i}$ be the profile of individual $i$. Individual $i$ receives fuzzy "utility," if the collective judgment set $q$ is collectively accepted, given by the formula $u_{i}(q)=\eta\left(\tilde{d}_{i}\left(p_{i}, q\right)\right) \in[0,1]$, where $\eta:[0,1] \rightarrow$ $[0,1]$ is the strong fuzzy negation (see Nguyen and Walker [15]) that satisfies the following:

(i) $\eta(0)=1, \eta(1)=0$,

(ii) $\eta$ is nonincreasing, and

(iii) $\eta(\eta(x))=x$.

For simplicity sake assumes that $\eta(x)=1-x$. Now

$$
u_{i}(q)=1-\tilde{d}_{i}\left(p_{i}, q\right)
$$

implies that

$$
\sum_{\forall i} u_{i}(q)+\sum_{\forall i} \tilde{d}_{i}\left(p_{i}, q\right)=n .
$$

Choosing a collective judgment set $q$ which minimizes $\sum_{\forall i} \tilde{d}_{i}\left(p_{i}, q\right)$ is, therefore, equivalent to choosing $q$ which maximizes $\sum_{\forall i} u_{i}(q)$, that is, the sum of individual utilities. Therefore, optimization problems in Table 3 can be viewed as social utility maximization problems.

\section{Conclusion}

Fuzzy aggregation procedures are useful in constructing optimal fuzzy social preference aggregation functions as already illustrated in the previous discussions. Finding such optimal fuzzy preference structures could have great applications in social choice theory by bringing it closer to reality. The real challenge is to construct aggregation procedures that satisfy desirable social properties and at the same time do not violate collective rationality. Authors believe that modeling impossibility theorems in fuzzy setting will have tremendous applications in the field of belief merging and judgment aggregation.

\section{Acknowledgment}

The present version of the paper owes much to the precise and kind remarks of the learned referees.

\section{References}

[1] F. Dietrich, "A generalised model of judgment aggregation," Social Choice and Welfare, vol. 28, no. 4, pp. 529-565, 2007.

[2] F. Dietrich and C. List, "Where do preference come from?" International Journal of Game Theory. In press.

[3] C. List, "Free will, determinism and the possibility of doing otherwise," working paper, London School of Economics, 2011, http://personal.lse.ac.uk/list/PDF-files/Freewill.pdf.
[4] I. Beg and N. Butt, (Im)Possibility Theorems in Fuzzy Framework, Critical Review, vol. 4, Society for Mathematics of Uncertainity, 2010.

[5] P. Manzini and M. Mariotti, "Moody choice," working paper University of St. Andrews, 2012.

[6] C. List and P. Pettit, "Aggregating sets of judgments: an impossibility result," Economics and Philosophy, vol. 18, pp. 89-110, 2002.

[7] C. List and P. Pettit, Group Agency: The Possibility, Design and Status of Corporate Agents, Oxford University Press, Oxford, UK, 2011.

[8] K. J. Arrow, Social Choice and Individual Values, John Wiley \& Sons, New York, NY, USA, 2nd edition, 1963.

[9] A. Sen, Collective Choice and Social Welfare, Holden Day, San Francisco, Calif, USA, 1970.

[10] F. Dietrich, "Judgment aggregation: (Im)possibility theorems," Journal of Economic Theory, vol. 126, no. 1, pp. 286-298, 2006.

[11] M. Van Hees, "The limits of epistemic democracy," Social Choice and Welfare, vol. 28, no. 4, pp. 649-666, 2007.

[12] G. Pigozzi, "Belief merging and the discursive dilemma: an argument-based account to paradoxes of judgment aggregation," Synthese, vol. 152, no. 2, pp. 285-298, 2006.

[13] C. List, "Group knowledge and group rationality: a judgment aggregation perspective," Episteme, vol. 2, no. 1, pp. 25-38, 2005.

[14] C. A. Claussen and O. Roisland, "Collective economic decisions and the discursive paradox," Norges Bank Working paper, 2005.

[15] H. T. Nguyen and E. Walker, Fuzzy Logic, Chapman \& Hall/CRC Press, 3rd edition, 2006.

[16] F. Dietrich and C. List, "Strategy-proof judgment aggregation," Economics and Philosophy, vol. 23, no. 3, pp. 269-300, 2007.

[17] J. I. Peláez and J. M. Doña, "LAMA: a linguistic aggregation of majority additive operator," International Journal of Intelligent Systems, vol. 18, no. 7, pp. 809-820, 2003.

[18] R. R. Yager, "On ordered weighted averaging operators in multi-criterion decision making," IEEE Transactions on Systems, Man and Cybernetics, vol. 18, no. 1, pp. 183-190, 1988.

[19] C. List and P. Pettit, "On the many as one: a reply to Kornhauser and Sager," Philosophy and Public Affairs, vol. 33, no. 4, pp. 377-390, 2005. 

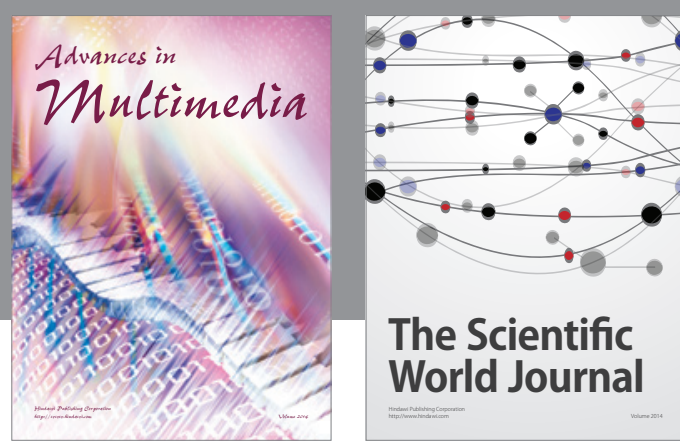

The Scientific World Journal
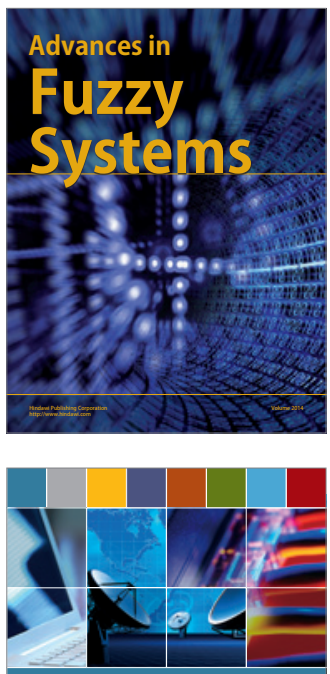

Computer Networks and Communications
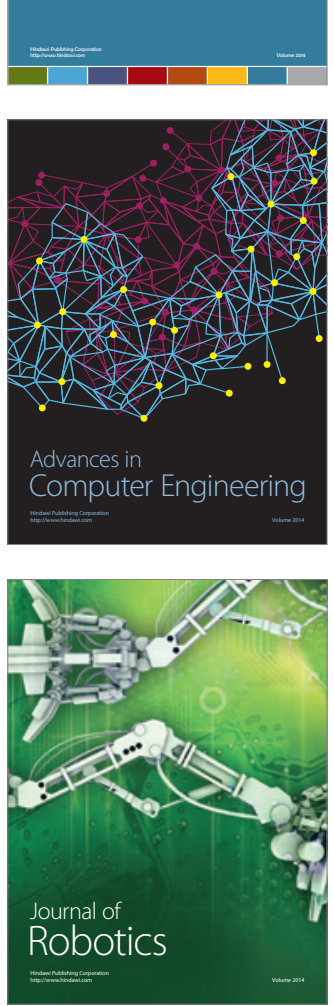
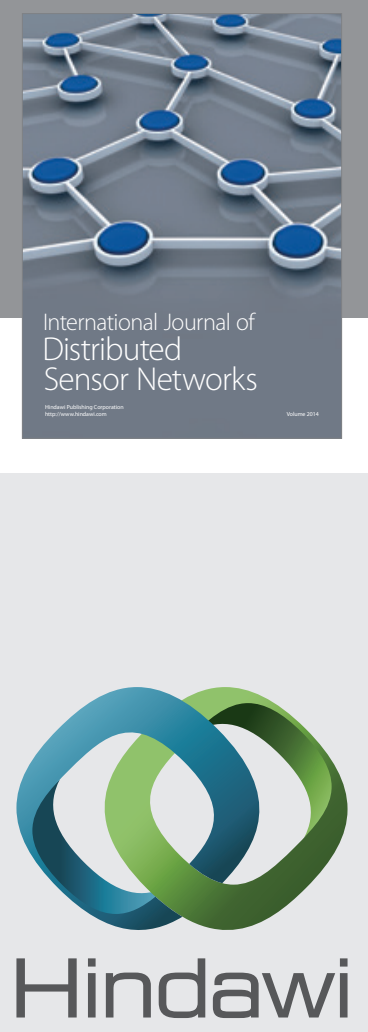

Submit your manuscripts at

http://www.hindawi.com
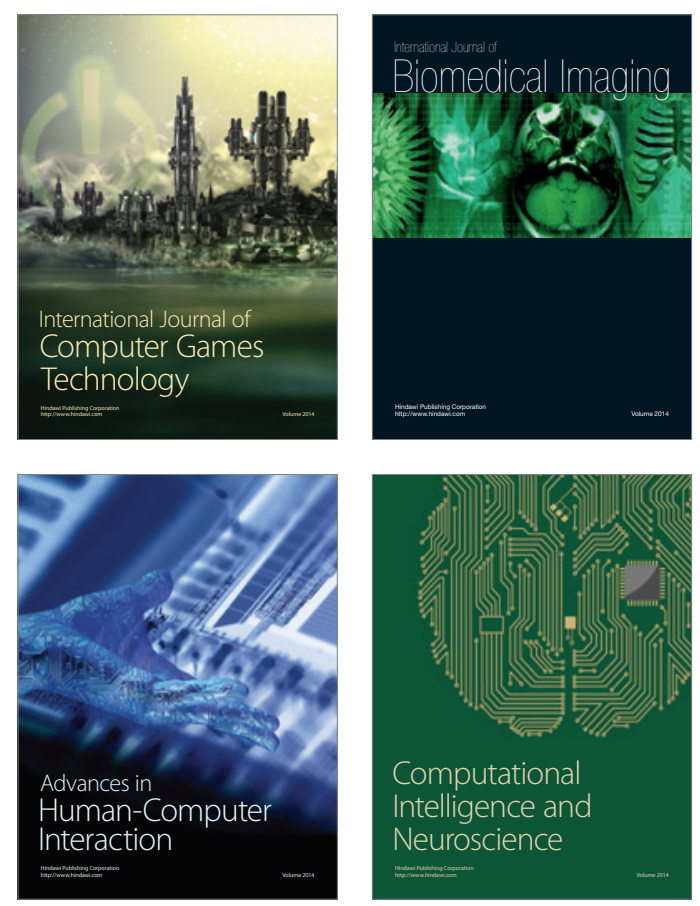
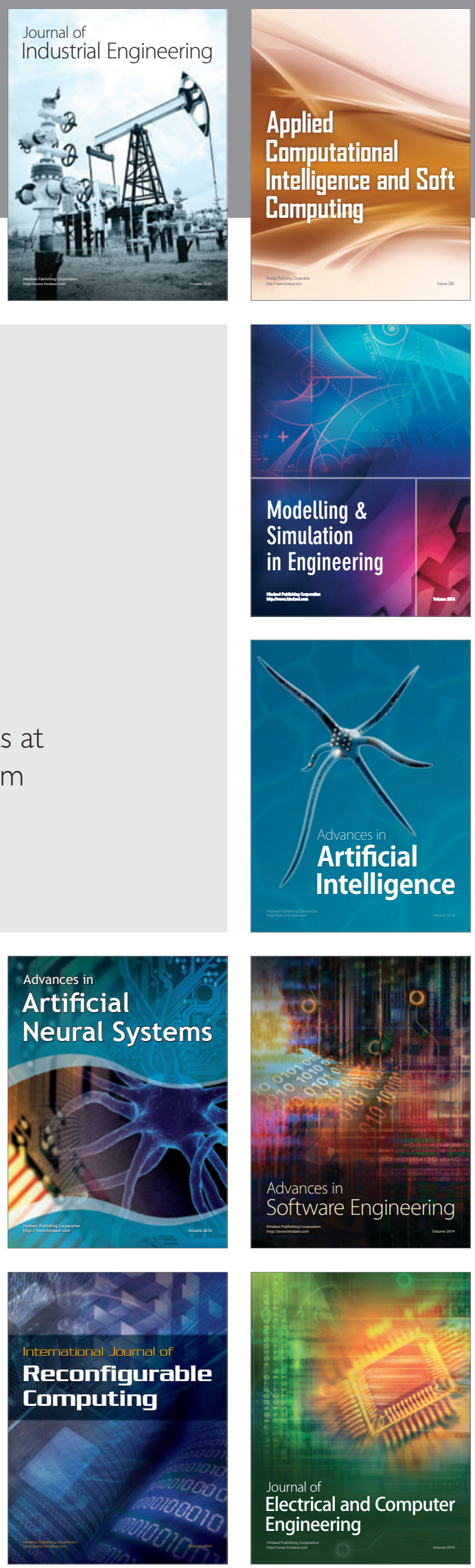